\title{
Docking studies to explore novel lead molecules for human spleen tyrosine kinase
}

involved in chronic lymphocytic leukemia

Naveen kadaari,* Dibyabhaba Pradhan, Muni kumar and Amineni Umamaheswari**

Bioinformatics Centre, Department of Bioinformatics, SVIMS University, Tirupati, PIN 517507.

* Presenting Author; ** Corresponding Author; Email: svims.btisnet@nic.in

1.B-cell chronic lymphocytic leukemia (CLL) is the most prevalent B-cell malignancy in adults.

2.Spleen tyrosine kinase (SYK) plays a pivotal role for B-lymphocyte development and maturation in B-cell receptor (BCR) signaling pathway

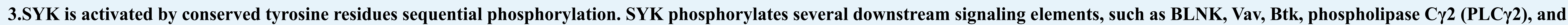
growth factor receptor binding proteins.

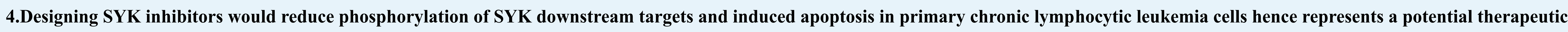
target for CLL.

The present study is directed towards finding novel inhibitors of SYK through ligand based virtual screening.

\section{Materials \&imethods}

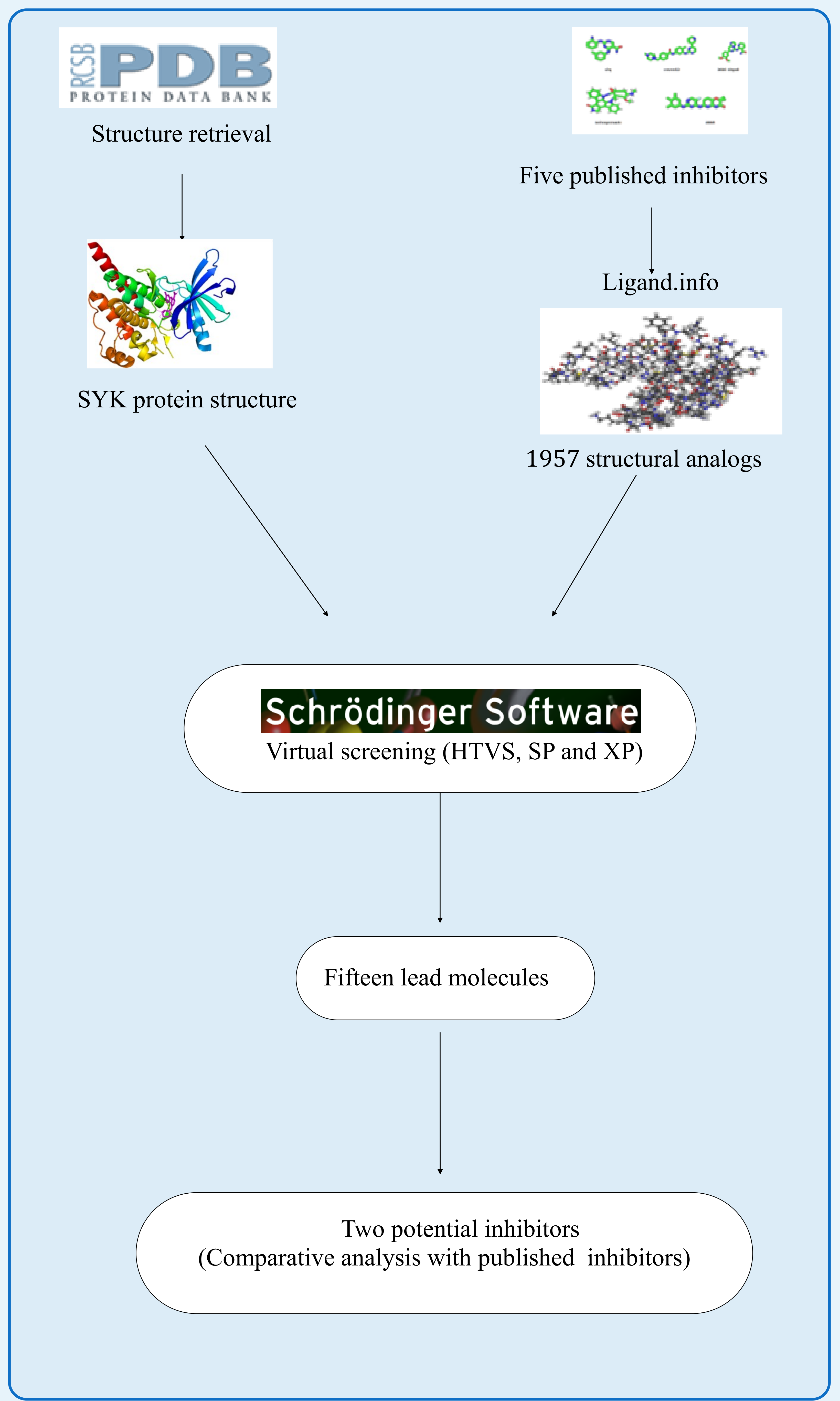

conclusion
Results \& discussion

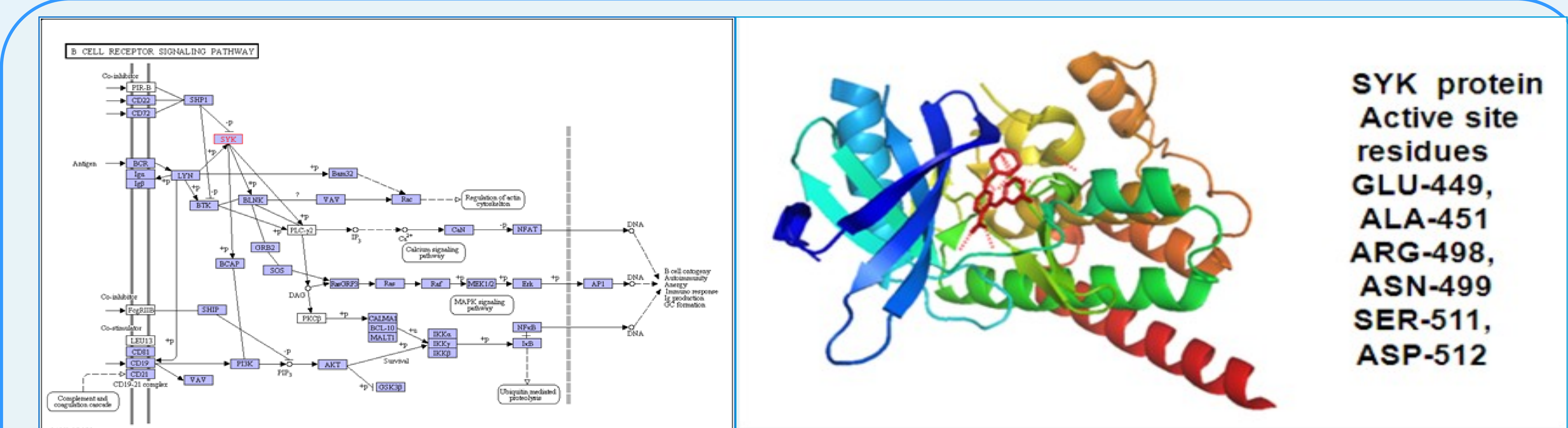

Figure1:SYK protein involved in B- cell receptor signalling Pathway

Figure2:Human SYK protein X-ray crystal structure with p5c ligand
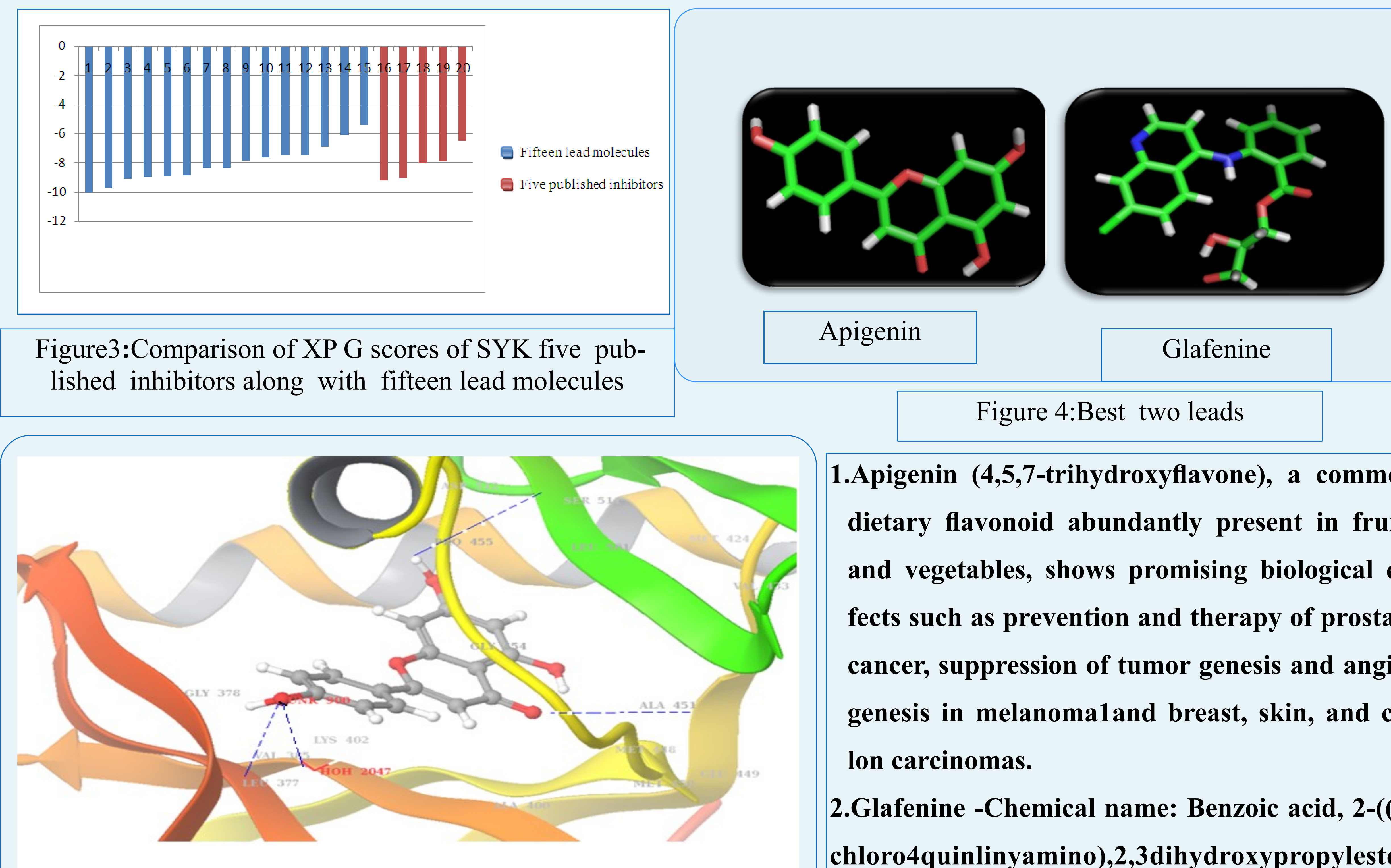

Figure 5:Lead ' 1 ' molecule (apigenin) forming 4 Hydrogen bond with SYK protein residues and least docking score-(-10.05 kcal $/ \mathrm{mol})$. Hydrogen bonds are Leu-377,Ala-451,Asp-512W-2047
1.Apigenin (4,5,7-trihydroxyflavone), a common dietary flavonoid abundantly present in fruits and vegetables, shows promising biological effects such as prevention and therapy of prostate cancer, suppression of tumor genesis and angiogenesis in melanoma1and breast, skin, and colon carcinomas.

2.Glafenine -Chemical name: Benzoic acid, 2-((7chloro4quinlinyamino),2,3dihydroxypropylester. An anthranilic acid derivative with analgesic properties used for the relief of all types of pain.

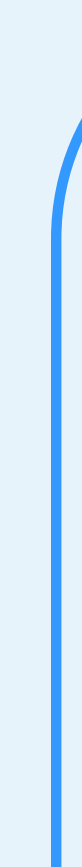

\begin{abstract}
Fifteen lead molecules with good binding affinity to SYK were identified and compared with binding affinities and orientations of five published inhibitors.

Apigenin (-10.05 Kcal/mol) and Glafenine (-9.72 Kcal/mol) endeavored better binding affinities, hydrogen bonding network and Van der Waal interactions with active site residues compared to published inhibitors.Thus, Apigenin and Glafenine would be valuable for designing novel inhibitors against CLL, if synthesized and tested in vitro and in vivo.
\end{abstract}

\section{Acknowledgement}

Authors are highly thankful to DBT, Ministry of science and technology, Govt. of India for supporting research activi-ties at SVIMS Bioinformatics centre. We are also thankful to Dr. B.Vengamma, Director, SVIMS, for her constant en-couragement and support. 